\title{
Actualización en la comprensión y manejo de la intoxicación por acetaminofén
}

\section{Update on the Understanding and Management of Intoxication by Acetaminophen}

Recepción: 22/01/2018 | Aceptación: 27/10/2018

\author{
Rafael Gustavo Castellanos ${ }^{\mathrm{a}}$ \\ GARZÓN \\ Hospital San Ignacio, Colombia \\ Elkin René Barrios Peralta \\ Hospital San Ignacio, Colombia \\ Alberto Vides \\ Hospital San Ignacio, Colombia \\ María Francisca Olarte \\ Universidad del Rosario, Colombia
}

a Correspondencia:

r_castellanos@javeriana.edu.co

Cómo citar: Castellanos Garzón RG, Barrios Peralta ER, Vides A, Olarte MF. Actualización en la comprensión y manejo de la intoxicación por acetaminofén. Univ. Med. 2018;59(4). doi: https://doi.org/10.11144/Javeriana.umed59-4.acet

\section{RESUMEN}

La intoxicación por acetaminofén es una de las causas más frecuentes de falla hepática aguda fulminante y una de las principales causas de atención en los servicios de urgencias, por intoxicación debida a medicamentos. Recientemente, se han registrado avances en la comprensión fisiopatológica, diagnóstico y tratamiento de esta entidad clínica. Los mecanismos de estrés oxidativo y daño del ADN mitocondrial explican los daños producidos en esta intoxicación. En esta revisión se presentan aspectos referentes a la epidemiología, mecanismos fisiopatológicos, diagnóstico y tratamiento de la intoxicación por acetaminofén.

\section{Palabras clave}

sobredosis de droga; acetaminofén; enfermedad hepática inducida por drogas y medicamentos.

\begin{abstract}
Acetaminophen poisoning is one of the most frequent causes of acute fulminant hepatic failure and one of the main causes of care in emergency services due to drug intoxication. Recent progress has been made in the pathophysiological understanding, diagnosis and treatment of this clinical entity. The mechanisms of oxidative stress and mitochondrial DNA damage explain the damage caused by this intoxication. This review presents aspects related to the epidemiology, pathophysiological mechanisms, diagnosis and treatment of acetaminophen intoxication.

Keywords

drug overdose; acetaminophen; hepatotoxicity.
\end{abstract}

\section{Introducción}

El acetaminofén fue sintetizado por primera vez a finales del siglo XIX. Sin embargo, solo después de la década de 
los cincuenta se convirtió en uno de los medicamentos analgésicos y antipiréticos de venta libre más utilizados en todo el mundo $(1,2)$; más del 90\% de la población reconoce su utilidad para el manejo del dolor, y un 50\%, para el manejo de la fiebre (3).

El acetaminofén es un medicamento considerado seguro, por su amplia ventana terapéutica; en consecuencia, el riesgo de sobredosis por una toma inadecuada disminuye. A pesar de ello, las intoxicaciones por acetaminofén son relativamente frecuentes en la población general (4). La intoxicación puede conducir a alteraciones severas del estado general, principalmente un grave daño hepático. En Estados Unidos, la intoxicación por acetaminofén es causante del 39\% de falla hepática aguda, es decir, la más frecuente (5).

En la actualidad, el estudio de la intoxicación por acetaminofén (IA) es un campo activo de investigación, dado el incremento en la producción bibliográfica sobre este tema (6). El objetivo de este artículo es revisar aspectos relacionados con la epidemiología, la fisiopatología, el diagnóstico y el manejo de la IA.

\section{Epidemiología}

Un estudio realizado en España entre 2005 y 2010 encontró que la incidencia de la IA es de 2 casos por cada 10.000 personas, con una tendencia al aumento, probablemente, debido a la mayor formulación, disponibilidad del medicamento y sensibilización hacia la detección de casos de intoxicación (7).

En Estados Unidos, la IA produce más de 400.000 visitas a los servicios de urgencias, genera costos directos de atención por más de mil millones de dólares por año (8), con una prevalencia de admisiones a servicios de urgencias del 5,8\% de los casos relacionados con intoxicación por medicamentos (8).

Un estudio realizado en Chile documentó que las intoxicaciones por medicamentos corresponden al 0,5\% del total de admisiones en los servicios de urgencias, y de ellas, el 6,8\% son debidas a intoxicación por acetaminofén
$(9,10)$, es decir, la frecuencia de la intoxicación se presenta de manera similar en Chile y Estados Unidos.

Factores de riesgo asociados

Algunos estudios han revisado los factores asociados con la presentación de la IA. La mayoría de los casos se da cuando el acetaminofén se prescribe en combinación con otros principios activos, especialmente opioides. Alrededor del $50 \%$ de estos casos no son intencionales y se relacionan con una sobredosificación para mejorar el control sintomático del dolor (11). Otro estudio muestra que el 30\% de los casos de IA se ha detectado con el suministro del acetaminofén sin combinaciones, y el $17 \%$, en combinación con difenhidramina (12). La edad es un factor relacionado con la frecuencia aparición de casos, especialmente aquellos que son intencionales (edad entre los 14 y los 24 años); sin embargo, una edad superior a los 40 años es un factor de riesgo plenamente identificado para una mayor mortalidad, es decir, aunque la frecuencia de intoxicaciones es mayor en la adolescencia, la severidad del cuadro clínico es superior en los adultos (13). Estos aspectos deben ser tenidos en cuenta para la identificación y manejo de la IA.

Otros factores de riesgo parecen estar relacionados con las causas de prescripción del medicamento. En un estudio de casos y controles se encontró que el dolor de origen odontológico se constituye en un factor de riesgo para la sobredosis por acetaminofén (OR: 12,6; IC95\%: 4,2-47,6) (14). Así mismo, hay factores que influyen en el riesgo de presentación de una IA y en el desarrollo de hepatotoxicidad: el consumo crónico de alcohol, la ingesta de medicamentos que incrementan la actividad de la CYP2E1, la malnutrición, el embarazo y la enfermedad hepática crónica (15).

Una de las complicaciones de la IA es la falla hepática fulminante, caso en el cual estaría indicado un trasplante hepático. Recientemente se publicaron los resultados de un estudio multicéntrico en Europa en el cual se encontró 
que la IA es causa del $20 \%$ de los casos de falla hepática aguda que requirieron trasplante hepático (16).

\section{Fisiopatología}

La principal vía de exposición al acetaminofén es la digestiva. En adultos se ha encontrado que dosis superiores a los $10 \mathrm{~g}$ conducen a la aparición de toxicidad, y superiores a $20 \mathrm{~g}$ suelen conducir a la mortalidad $(17,18)$. La velocidad de absorción del medicamento depende directamente de la velocidad de vaciamiento gástrico; la concentración máxima se alcanza a los 90 min luego de su ingesta, en concentraciones terapéuticas $(5-20 \mu \mathrm{g} / \mathrm{ml})$ no se presenta unión a proteínas plasmáticas, pero sí a concentraciones superiores (entre un 20\% y un 50\%) (19). A dosis tóxicas, las vías de conjugación habituales del acetaminofén se saturan; por consiguiente, se metaboliza principalmente por la CYP2E1 y se incrementan los niveles del NAPQI (N-acetil-para benzoquinoneimina), metabolito altamente tóxico, reactivo y con unión a muchos compuestos intracelulares, y al no poder ser eliminado por saturación de los mecanismos naturales de detoxificación (glutatión peroxidasa) aparece el daño oxidativo (20).

Varios estudios han estudiado los mecanismos por medio de los cuales se produce el daño en la IA. El principal daño es hepático, teniendo en cuenta que la principal vía metabólica del medicamento se realiza en el hígado (18). Generalmente, el daño hepático y renal se produce en pacientes que presentan niveles mayores a $100 \mu \mathrm{g} / \mathrm{ml}$ en menos de $12 \mathrm{~h}$ posteriores a la ingesta; raramente se da en pacientes con cifras inferiores a esta (21). El exceso de exposición de las células al NAPQI conduce a un deterioro progresivo de la función celular. Tres mecanismos han mostrado ser relevantes en el humano: inducción del estrés oxidativo, daño del ADN mitocondrial y la activación del factor de transcripción JNK (cinasas c-jun $\mathrm{N}$-terminal) (22).
Los fenómenos de estrés oxidativo en el daño hepático (y de otros tejidos) inducidos por acetaminofén son el mecanismo más estudiado y que parece explicar en su mayoría el cuadro clínico que se presenta en la IA (23). En un modelo animal en ratones se determinó la importancia de algunas enzimas implicadas en el desarrollo del estrés oxidativo. Los ratones que no expresan la superóxido dismutasa o la glutatión peroxidasa muestran una sobrevida de $50 \mathrm{~h}$, menor que aquellos que sí las expresan luego de la intoxicación por acetaminofén (24). La superóxido dismutasa y glutatión peroxidasa son enzimas directamente relacionadas con el control y la severidad del daño de los tejidos mediados por oxidación $(25,26)$. En casos de IA se presenta una reducción de la glutationización de las proteínas y un aumento del calcio intracelular; estos procesos son marcadores de la alteración de la función antioxidativa en el hepatocito (27). Estos procesos son en parte mediados por el canal TRPM2 (receptor transitorio potencial de melanostatina 2) del hepatocito (28). La inducción de la función y expresión de los canales TRPM2 están relacionados con procesos de alteración y muerte del hepatocito y carcinogénesis (29).

El daño del ADN mitocondrial es otro mecanismo fisiopatológico implicado en los daños de la IA. La formación de poros en la membrana mitocondrial conduce a una alteración del ADN (30). Estas modificaciones llevan a una alteración en los niveles de calcio y magnesio que alteran la homeostasis mitocondrial (31). Estos mecanismos hacen pensar que existen mecanismos moleculares de daño mitocondrial que sirven como marcadores del daño inducido por la IA.

Existe controversia sobre el efecto de la activación del JNK en la intoxicación hepática (32). Varios estudios muestran que la activación de la JNK explica el daño hepático en pacientes con IA (33). La vía de las JNK responde a estímulos de estrés oxidativo y se encuentra relacionada con varias enfermedades crónicas y degenerativas $(34,35)$. Dosis bajas de acetaminofén están relacionadas con una disfunción mitocondrial y activación reversible 
de la vía JNK; sin embargo, dosis altas compatibles con intoxicación se relacionan con el desarrollo de modificaciones irreversibles de la función mitocondrial y activación permanente de la vía JNK, que conduce a la muerte del hepatocito (36).

\section{Diagnóstico}

El diagnóstico debe tener en cuenta la clínica, la dosis tóxica y el resultado del análisis de laboratorio. El cuadro clínico suele presentarse en las primeras $24 \mathrm{~h}$ luego de la ingestión (sin embargo, algunos pacientes no presentan sintomatología inicial); la dosis tóxica en casos agudos en niños está entre 150 y $200 \mathrm{mg} / \mathrm{kg}$, y en adultos, entre 6 y $7 \mathrm{~g}$ con niveles superiores a los $150 \mu \mathrm{g} / \mathrm{ml}$ a las $4 \mathrm{~h}$, o superiores a los $18 \mu \mathrm{g} /$ $\mathrm{ml}$ a las $16 \mathrm{~h}$, y según el nomograma de Rumack Matthew se encontrarán en riesgo de desarrollar hepatotoxicidad (21).

En las primeras $24 \mathrm{~h}$ de la toxicidad aguda hay una elevación leve a moderada de las transaminasas hepáticas y síntomas como la epigastralgia, náusea y vómito (37). Luego de las $24 \mathrm{~h}$ y hasta $72 \mathrm{~h}$ se hacen evidentes signos de la hepatotoxicidad; 3 a 4 días después de la ingesta se presenta la falla hepática fulminante, y a los 5 días puede continuar la falla hepática o bien la falla multiorgánica fulminante o bien una recuperación del paciente (38).

Los retrasos en la absorción (debidos a los retrasos del vaciamiento gástrico) del acetaminofén pueden dar lugar a un diagnóstico errado de la severidad del cuadro clínico o a un retraso de su aparición. Condiciones clínicas $y$ el uso de medicamentos que retrasen el vaciamiento gástrico pueden demorar el tiempo de expresión sintomática de la toxicidad. Se ha sugerido un monitoreo periódico de los niveles de acetaminofén en casos en los cuales haya un retraso en la absorción del medicamento (39). Factores clínicos que alteran el vaciado gástrico, circulación enterohepática y la combinación de acetaminofén con opioides o antagonistas colinérgicos afectan de manera significativa la absorción del acetaminofén (40). Estos aspectos deben ser tenidos en cuenta por el clínico durante la atención del paciente. La figura 1 presenta las fases de la IA.

\section{Figura 1}

Fases clínicas de la IA

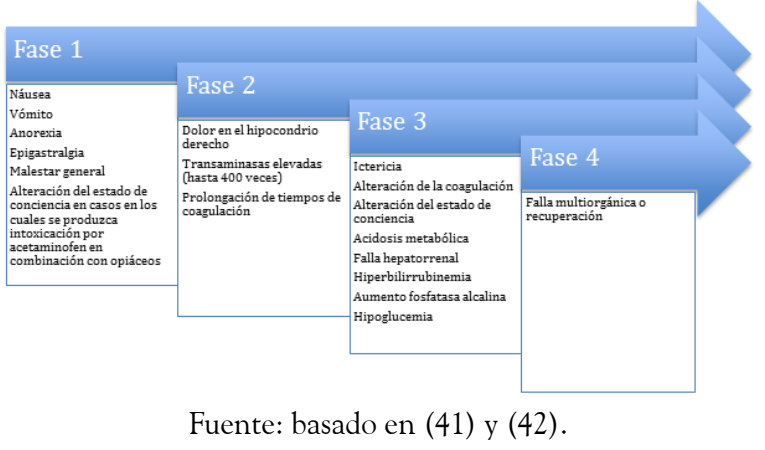

Cuando se evalúa a un paciente con historia de consumo crónico de dosis terapéuticas o supraterapéuticas, es de gran importancia, incluso ante la ausencia de síntomas, determinar la existencia de pruebas que sugieran hepatotoxicidad. En estos casos, niveles mayores a $10 \mu \mathrm{g} / \mathrm{ml}$ indican la necesidad de iniciar manejo contra la intoxicación, ya que en estos pacientes estos niveles podrían coexistir con daño hepático $(37,39)$. Una revisión extensa sobre el tema escapa a los objetivos de este artículo.

Se han investigado nuevos marcadores paraclínicos para el diagnóstico del daño hepático luego de la IA. Uno de ellos son los micro-RNA-122 extracelulares. En un estudio en niños con intoxicación por acetaminofén o sin esta se encontró que los micro-RNA extracelulares se identifican en sangre y en orina (43). En la actualidad se investiga, además, el papel del glutamato deshidrogenasa, molécula de daño renal-1, ADN mitocondrial y queratina 18, por su posible utilidad en el diagnóstico y pronóstico de pacientes con intoxicación por acetaminofén (44). Estas moléculas tienen como ventaja permitir la investigación en modelos animales (ratones), distinguir un aumento clínicamente relevante de la ALT y que son señales directas de daño hepático. Sin embargo, se requieren estudios que evalúen su real utilidad como prueba diagnóstica en el ámbito clínico (44). 
El nomograma de Rumack Mathew es una herramienta que se usa como guía de manejo después de una intoxicación aguda de acetaminofén. Se ha sugerido que el diagnóstico y manejo de la IA debe guiarse por los resultados del nomograma, pues la evaluación de la severidad del cuadro de acuerdo con la concentración de acetaminofén permite un enfoque terapéutico diferencial (figura 2) (45).

\section{Figura 2}

Nomograma de Rumack Mathew

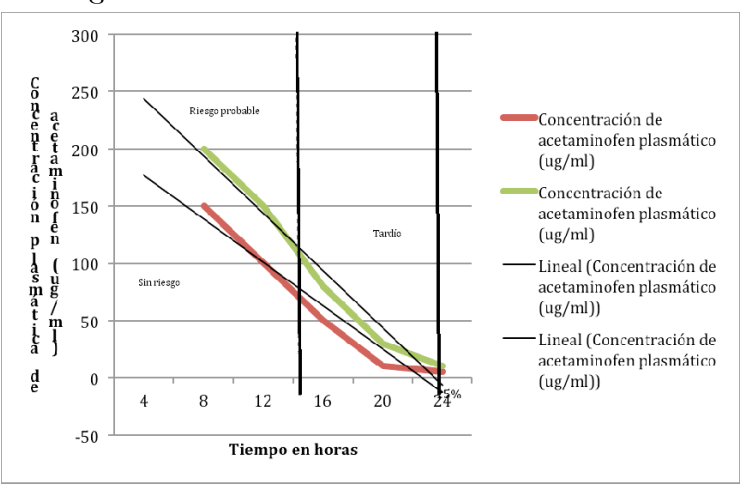

Fuente: adaptado de (45).

El nomograma ha mostrado una sensibilidad del $100 \%$ y una especificidad del $94 \%$ para la predicción de la hepatotoxicidad, es decir, se trata de una prueba diagnóstica con un buen rendimiento y que debe ser tenida en cuenta en la estadificación de riesgo de estos pacientes (46).

La presentación de algunas manifestaciones clínicas, especialmente aquellas relacionadas con la hepatotoxicidad están relacionadas con una mayor severidad del cuadro clínico. La presentación de una categoría de riesgo de toxicidad por acetaminofén en el nomograma, la elevación de los tiempos de protrombina, niveles de bilirrubina, niveles de creatinina y niveles de potasio sérico son algunos indicadores de severidad del cuadro clínico en los pacientes (47). La mortalidad se presenta en el 0,5\% de los casos (48).

La intoxicación por acetaminofén se puede también presentar con pancreatitis. El riesgo en estos pacientes es de hasta 3,11 veces mayor que en los pacientes de control sin intoxicación. Sin embargo, en pacientes de más de 30 años se presenta un riesgo mayor (49).
Es importante, dentro de la evaluación clínica de estos pacientes, tener en cuenta los criterios del King's College Hospital para la evaluación de la necesidad de trasplante hepático urgente $(\mathrm{pH}$ arterial $<7,3$ independiente del grado de encefalopatía), encefalopatía grado III o IV, tiempo de protrombina $>100 \mathrm{~s}$ y creatinina sérica $>3,4 \mathrm{mg} / \mathrm{dl}(301 \mu \mathrm{mol} / \mathrm{l})$ y el MELD score, que fija pronóstico y permite predecir la mortalidad a 3 meses (39).

\section{Tratamiento}

Las decisiones terapéuticas se basan, por supuesto, en los hallazgos de la evaluación clínica y paraclínica del paciente. Se recomienda iniciar el esquema de manejo asumiendo una intoxicación en pacientes que presenten niveles de acetaminofén en sangre superiores a $200 \mu \mathrm{g} /$ $\mathrm{ml} 4 \mathrm{~h}$ después de la ingesta o en aquellos que presenten elevación de las transaminasas con antecedente de ingesta de acetaminofén a dosis superiores a los $6 \mathrm{~g}$ en un día (50). La acetilcisteína se considera el antídoto y manejo de elección en pacientes con IA. En general, el manejo aceptado supone su administración en bolo a razón de $150 \mathrm{mg} / \mathrm{kg}$ diluidos en 100 a 200 $\mathrm{ml}$ de dextrosa al 5\%, posterior infusión de $50 \mathrm{mg} /$ $\mathrm{kg}$ en 0,5 1 de solución salina o dextrosa al 5\% en las siguientes $4 \mathrm{~h}$ y luego $100 \mathrm{mg} / \mathrm{kg}$ en $11 \mathrm{de}$ solución salina o dextrosa al 5\% (tabla 1) (21).

\section{Tabla 1}

Dosis de N-acetilcisteína

\begin{tabular}{|l|l|}
\hline Intravenoso & Oral \\
\hline - Diluir en SSN, dextrosa al & - $140 \mathrm{mg} / \mathrm{kg}$ como \\
$5 \%$ o agua estéril. & dosis de carga \\
- Iniciar $150 \mathrm{mg} / \mathrm{kg}$ en 200 & - $70 \mathrm{mg} / \mathrm{kg}$ cada 4 \\
ml durante $15-60 \mathrm{~min}$ & horas por 17 dosis \\
- Luego $50 \mathrm{mg} / \mathrm{kg}$ en 500 & \\
ml durante $4 \mathrm{~h}$ & \\
- Continuar $100 \mathrm{mg} / \mathrm{kg}$ en & \\
$1000 \mathrm{ml}$ durante $16 \mathrm{~h}$ & \\
\hline
\end{tabular}

Fuente: basado en (51).

Algunos autores han mostrado que, a pesar de ser el régimen predominante en el manejo de la IA, no existe evidencia científica sólida 
que demuestre que se trata del mejor esquema de manejo (51). En ese sentido, esquemas más cortos o largos podrían ser igualmente válidos. Existen esquemas de manejo oral e intravenoso que son presentados en la tabla 1.

Uno de los problemas relacionados con el uso de la acetilcisteína es la incidencia de reacciones adversas. En un estudio retrospectivo se encontró que el $46 \%$ de los pacientes desarrollan alguna reacción adversa, siendo el vómito, erupción cutánea, prurito, dolor torácico, broncoespasmo y tos, los más frecuentes (administración intravenosa). En el 24\% de los pacientes estas reacciones adversas poseen una intensidad moderada a severa (52). Se ha reportado que a concentraciones mayores de 100 $\mu \mathrm{g} / \mathrm{ml}$ en sangre del acetaminofén se produce un menor riesgo de reacciones anafilactoides con el uso de la acetilcisteína (OR: 0,19; IC 95\%: 0,10-0,37) (53).

En un modelo animal se encontró que la coadministración de acetaminofén (600 mg) y acetilcisteína $(600 \mathrm{mg})$ reduce el desarrollo de la IA (54). Por este motivo es posible que en poblaciones de riesgo podría realizarse la coadministración de estos medicamentos para prevenir los casos de intoxicación; sin embargo, se requiere el desarrollo de evidencia científica en seres humanos, con el fin de determinar si esta coadministración es útil o no.

En un ensayo clínico se comparó el esquema habitual de manejo con acetilcisteína contra un esquema corto ( $12 \mathrm{~h}$ de duración). Los dos esquemas mostraron una eficacia similar; pero el esquema corto produjo menos vómito (OR: 0,26; IC 97,5\%: 0,13-0,52) y reacciones anafilácticas (OR: 0,23; IC 97,5\%: 0,12-0,43) (55). Se reportó un caso en el cual un esquema extenso a dosis de $420 \mathrm{mg} / \mathrm{kg} /$ día durante 7 días, seguido por 5 días a razón de $50 \mathrm{mg} / \mathrm{kg}$ cada 4 h permitió, luego de la presentación falla hepática severa secundaria a IA, la recuperación del paciente sin secuelas hepáticas aparentes (56). Fijar la dosis adecuada de acetilcisteína es trascendental. La sobredosis de acetilcisteína produce efectos negativos sobre la economía corporal entre ellos el edema cerebral y convulsiones los cuales pueden conducir a la muerte (57).
El uso de acetilcisteína durante más de 24 horas se considera un esquema extendido y es susceptible de una mayor tasa de reacciones adversas (58). Este, sin embargo, es un tema controversial. Existen reportes de casos que muestran que a dosis altas no necesariamente se produce una mayor proporción de efectos adversos (59).

La metionina es otro medicamento utilizado en el manejo de la intoxicación por acetaminofén por sus efectos para reducir la hepatotoxicidad. La acetilcisteína es la más utilizada, la metionina se utiliza en menor medida y su administración por vía oral ha sido relacionada con un incremento en la proporción de pacientes con vómito; sin embargo, su perfil de seguridad no cuenta con suficiente evidencia científica (60).

Habitualmente, la reducción de niveles de transaminasas hepáticas, un nivel cercano a cero de los niveles de acetaminofén en sangre, asî como un examen clínico normal son indicadores para la suspensión del tratamiento. Un radio menor de 0,4 en los niveles de AST/ALT son indicadores que permiten tomar una decisión clínica objetiva en estos pacientes y permite suspender el manejo con acetilcisteína (61).

Los pacientes que presentan mayor nivel de acetaminofén en sangre luego de las primeras 7 h luego de la ingestión están relacionadas con una mayor hepatotoxicidad y un mayor requerimiento de acetilcisteína (62).

\section{Hepatoprotección}

Existen diferentes esfuerzos por la búsqueda de agentes con función hepatoprotectora en casos de IA. El control de la oxidación mitocondrial es un blanco terapéutico que puede proteger el tejido hepático (30). En un modelo animal de intoxicación se encontró que los extractos de Cytrus hystrix (lima kafir) y Cucurbita maxima (una especie de calabaza) mejoran los marcadores de función hepática y la evaluación histológica del hígado (63).

En un modelo animal se encontró que la administración de metformina ejerce efectos protectores sobre la presentación del daño 
hepático inducido por el acetaminofén. Este efecto es mediado por la inhibición del proceso de activación del JNK, que conduce al daño hepático (64). Estudios clínicos deberán explorar si el uso de la metformina puede indicarse en los casos de IA.

En pacientes con IA se han encontrado niveles elevados de agentes oxidantes y disminución de marcadores de actividad y capacidad antioxidante. Teniendo en cuenta estos hallazgos se ha sugerido que las vitaminas $C_{\text {y }} E$ pueden ser útiles en el manejo de pacientes con IA (65).

La acetilcarnitina también ha sido evaluada por sus efectos hepatoprotectores. Se ha encontrado que reduce las especies de oxígeno reactivo, reduce los marcadores de daño hepático e induce el funcionamiento de las enzimas protectoras del daño oxidativo a nivel hepático (66).

\section{Conclusiones}

La IA es un problema que se presenta con frecuencia. Su proceso fisiopatológico se encuentra relacionado con el desarrollo de daño celular mediado por estrés oxidativo. Dado que se trata de un medicamento de libre acceso para los pacientes, limitar el número de tabletas en las presentaciones comerciales de acetaminofén ha mostrado reducir los casos de admisiones en unidades de cuidado hepático en el Reino Unido (de 30 tabletas a 14), por la dificultad que representa para las personas la consecución del medicamento (67).

El diagnóstico de la intoxicación se basa en las manifestaciones clínicas, dosis tóxica y paraclínicos; en la actualidad, se encuentran en curso nuevas investigaciones que permitan una detección temprana de los órganos afectados.

El uso de la acetilcisteína es el manejo habitual para la intoxicación. Se proponen tratamientos alternos que podrían ayudar al control de la hepatotoxicidad.

\section{Referencias}

1. Spooner JD, Harvey JG. The history of paracetamol and usage of paracetamol. J Int Med Res 1976; 4 (supl 1):1-6.

2. Bertolini A, Ferrari A, Ottani A, Guerzoni S, Tacchi R, Leone S. Paracetamol: New vistas of an old drug. CNS Drug Rev. 2006;12(3-4):250-75.

3. Gilbertson RJ, Harris E, Pandey SK, Kelly P, Myers W. Paracetamol use, availability, and knowledge of toxicity among British and American adolescents. Arch Dis Child. 1996; 75(3):194-8.

4. Graham GG, Davies MJ, Day RO, Mohamudally A, Scott KF. The modern pharmacology of paracetamol: Therapeutic actions, mechanism of action, metabolism, toxicity and recent pharmacological findings. Inflammopharmacology. 2013 Jun;21(3):201-32.

5. Ostapowicz G, Fontana RJ, Schiødt FV, Larson A, Davern TJ, Han SH, et al. Results of a prospective study of acute liver failure at 17 tertiary care centers in the United States. Ann Intern Med. 2002; 137(12):947-54.

6. Zyoud SH, Al-Jabi SW, Sweileh WM. Worldwide research productivity of paracetamol (acetaminophen) poisoning: A bibliometric analysis (2003-2012). Hum Exp Toxicol. 2015; $34(1): 12-23$.

7. Tong HY, Medrano N, Borobia AM, Martínez AM, Martín J, Ruiz JA, et al. V Hepatotoxicity induced by acute and chronic paracetamol overdose in adults. Where do we stand? Regul Toxicol Pharmacol. 2015; 72(2):370-8.

8. Altyar A, Kordi L, Skrepnek G. Clinical and economic characteristics of emergency department visits due to 
acetaminophen toxicity in the USA. BMJ Open. 2015;5(9):e007368.

9. Major JM, Zhou EH, Wong HL, Trinidad JP, Pham TM, Mehta H, et al. Trends in rates of acetaminophenrelated adverse events in the United States. Pharmacoepidemiol Drug Saf. 2016;25(5):590-8.

10. Aguilera P, Garrido M, Lessard E, Swanson J, Mallon WK, Saldias F, et al. Medication Overdoses at a Public Emergency Department in Santiago, Chile. West J Emerg Med. 2016; 17(1):75-80.

11. Blieden M, Paramore LC, Shah D, Ben-Joseph R. A perspective on the epidemiology of acetaminophen exposure and toxicity in the United States. Expert Rev Clin Pharmacol. 2014;7(3):341-8.

12. Serper M, Wolf MS, Parikh NA, Tillman H, Lee WM, Ganger DR. Risk factors, clinical presentation, and outcomes in overdose with acetaminophen alone or with combination products: results from the acute liver failure study group. J Clin Gastroenterol. 2016;50(1):85-91.

13. Schmidt LE. Age and paracetamol self-poisoning. Gut. 2005;54(5):686-90.

14. Vogel J, Heard KJ, Carlson C, Lange C, Mitchell G. Dental pain as a risk factor for accidental acetaminophen overdose: a case-control study. Am J Emerg Med. 2011;29(9):1125-9.

15. Bunchorntavakul C, Reddy KR. Acetaminophen-related

hepatotoxicity. Clin Liver Dis. 2013;17(4):587-607.

16. Gulmez SE, Larrey D, Pageaux GP, Bernuau J, Bissoli F, Horsmans Y, et al. Liver transplant associated with paracetamol overdose: results from Cseven-country SALT study. Br J Clin Pharmacol. 2015;80(3):599-606.
17. Bertolini A, Ferrari A, Ottani A, Guerzoni S, Tacchi R, Leone S. Paracetamol: New vistas of an old drug. CNS Drug Reviews 2006;12(3-4):250-75.

18. Jóźwiak-Bebenista M, Nowak JZ. Paracetamol: mechanism of action, applications and safety concern. Acta Pol Pharm. 2014;71(1):11-23.

19. Raffa RB, Pergolizzi JV Jr, Taylor R Jr, Decker JF, Patrick JT. Acetaminophen (paracetamol) oral absorption and clinical influences. Pain Pract. 2014;14(7):668-77.

20. Téllez AM. Aspectos toxicológicos del acetaminofén [internet]. [Citado 2017 abr 10] Disponible en: http://clinicalevidence.pbworks.co $\mathrm{m} / \mathrm{w} / \mathrm{file} / \mathrm{fetch} / 78806546 /$ Aspectos

21. Bateman DN. Changing the management of paracetamol poisoning. Clin Ther. 2015;37 (9):2135-41.

22. Jaeschke $H$. Acetaminophen: Dose-dependent drug hepatotoxicity and acute liver failure in patients. Dig Dis. 2015;33(4):464-71.

23. Yuan L, Kaplowitz N. Mechanisms of drug-induced liver injury. Clin Liver Dis. 2013;17(4):507-18.

24. Lei $\mathrm{XG}$, Zhu JH, McClung JP, Aregullin M, Roneker CA. Mice deficient in $\mathrm{Cu}, \mathrm{Zn}$-superoxide dismutase are resistant to acetaminophen toxicity. Biochem J. 2006;399(3):455-61.

25. Inupakutika MA, Sengupta $S$, Devireddy AR, Azad RK, Mittler $R$. The evolution of reactive oxygen species metabolism. J Exp Bot. 2016;67 (21):5933-5943.

26. Mello T, Zanieri F, Ceni E, Galli A. Oxidative Stress in the Healthy and Wounded Hepatocyte: A Cellular Organelles Perspective. Oxid Med Cell Longev. 2016;2016:8327410. 
27. Yang X, Greenhaw J, Ali A, Shi Q, Roberts DW, Hinson JA, et al. Changes in mouse liver protein glutathionylation after acetaminophen exposure. J Pharmacol Exp Ther. 2012;340(2):360-8.

28. Kheradpezhouh E, Ma L, Morphett A, Barritt GJ, Rychkov GY. TRPM2 channels mediate acetaminopheninduced liver damage. Proc Natl Acad Sci U S A. 2014;111(8):3176-81.

29. Rychkov GY, Barritt GJ. Expression and function of TRP channels in liver cells. Adv Exp Med Biol. 2011;704:667-86.

30. Jaeschke H, Bajt ML. Intracellular signaling mechanisms of acetaminophen-induced liver cell death. Toxicol Sci. 2006;89(1):31-41.

31. Jaeschke $\mathrm{H}, \mathrm{McGill} \mathrm{MR}$, Ramachandran A. Oxidant stress, mitochondria, and cell death mechanisms in drug-induced liver injury: Lessons learned from acetaminophen hepatotoxicity. Drug Metab Rev. 2012;44(1):88-106.

32. Du K, Xie Y, McGill MR, Jaeschke H. Pathophysiological significance of c-jun $\mathrm{N}$-terminal kinase in acetaminophen hepatotoxicity. Expert Opin Drug Metab Toxicol. 2015;11(11):1769-79.

33. Hu J, Ramshesh VK, McGill MR, Jaeschke H, Lemasters JJ. Low Dose Acetaminophen Induces Reversible Mitochondrial Dysfunction Associated with Transient c-Jun N-Terminal Kinase Activation in Mouse Liver. Toxicol Sci. 2016;150(1):204-15.

34. Liu FC, Lee HC, Liao CC, Li AH, Yu HP. Tropisetron protects against acetaminophen-induced liver injury via suppressing hepatic oxidative stress and modulating the activation of JNK/ERK MAPK pathways. Biomed Res Int. 2016;2016:1952947.
35. Du K, Xie Y, McGill MR, Jaeschke H. Pathophysiological significance of $\mathrm{c}$-jun $\mathrm{N}$-terminal kinase in acetaminophen hepatotoxicity. Expert Opin Drug Metab Toxicol. 2015;11(11):1769-79.

36. $\mathrm{Hu} \mathrm{J}$, Ramshesh VK, McGill MR, Jaeschke H, Lemasters JJ. Low dose acetaminophen induces reversible mitochondrial dysfunction associated with transient c-Jun $\mathrm{N}$-terminal kinase activation in mouse liver. Toxicol Sci. 2016;150(1):204-15.

37. Mancipe LC, Fernández DC, Fernández DG. Intoxicación por acetaminofén. Rev Fac Med. 2010;18(2):221-7.

38. Janssen J, Singh-Saluja S. How much did you take?: Reviewing acetaminophen toxicity. Can Fam Physician. 2015 Apr;61(4):347-9.

39. Papazoglu C, Ang JR, Mandel M, Basak P, Jesmajian S. Acetaminophen overdose associated with double serum concentration peaks. J Community Hosp Intern Med Perspect. 2015;5(6):29589.

40. Hendrickson RG, McKeown NJ, West PL, Burke CR. Bactrian ("double hump") acetaminophen pharmacokinetics: a case series and review of the literature. J Med Toxicol. 2010;6(3):337-44.

41. Roldán T, López A. Intoxicación por acetaminofén en pediatría: Aproximación y manejo. Uni. Méd. 2012;53(1):56-67.

42. Yoon E, Babar A, Choudhary M, Kutner M, Pyrsopoulos N. Acetaminopheninduced hepatotoxicity: A comprehensive update. J Clin Transl Hepatol. 2016;4(2):131-42.

43. Yang $X$, Salminen WF, Shi Q, Greenhaw J, Gill PS, Bhattacharyya S, et al. Potential 
of extracellular microRNAs as biomarkers of acetaminophen toxicity in children. Toxicol Appl Pharmacol. 2015;284(2):180-7.

44. Vliegenthart AD, Antoine DJ, Dear JW. Target biomarker profile for the clinical management of paracetamol overdose. Br J Clin Pharmacol. 2015 Sep;80(3):351-62.

45. Bateman DN. Paracetamol poisoning: Beyond the nomogram. Br J Clin Pharmacol. 2015;80(1):45-50.

46. Chomchai S, Lawattanatrakul N, Chomchai C. Acetaminophen Psi Nomogram: A sensitive and specific clinical tool to predict hepatotoxicity secondary to acute acetaminophen overdose. J Med Assoc Thai. 2014;97(2):165-72.

47. Zyoud SH, Awang R, Sulaiman SA, Al-Jabi SW. Association between gastrointestinal manifestations following acetaminophen poisoning and outcome in 291 acetaminophen poisoning patients. Pharmacoepidemiol Drug Saf. 2010;19(5):511-7.

48. Hou YC, Lin JL, Huang WH, Weng CH, Lee SY, Hsu CW, et al. Outcomes of patients with acetaminophenassociated toxic hepatitis at a far east poison center. Springerplus. 2013 Dec 17;2:674.

49. Chen SJ, Lin CS, Hsu CW, Lin CL, Kao CH. Acetaminophen poisoning and risk of acute pancreatitis: A population-based cohort study. Medicine (Baltimore). 2015;94(29):e1195.

50. Heard KJ. Acetylcysteine for acetaminophen poisoning. $\mathrm{N}$ Engl J Med. 2008 Jul 17;359(3):285-92.

51. Wong A, Graudins A. $\mathrm{N}$-acetylcysteine regimens for paracetamol overdose: Time for a change? Emerg Med Australas. 2016;28(6):749-51.

52. Zyoud SH, Awang R, Syed Sulaiman SA, Sweileh WM, AlJabi SW. Incidence of adverse drug reactions induced by $\mathrm{N}$ acetylcysteine in patients with acetaminophen overdose. Hum Exp Toxicol. 2010;29(3):153-60.

53. Bateman DN, Carroll R, Pettie J, Yamamoto T, Elamin ME, Peart L, et al. Effect of the UK's revised paracetamol poisoning management guidelines on admissions, adverse reactions and costs of treatment. Br J Clin Pharmacol. 2014;78(3):610-8.

54. Owumi SE, Andrus JP, Herzenberg LA, Herzenberg LA. $\mathrm{Co}$-administration of $\mathrm{N}$-acetylcysteine and acetaminophen efficiently blocks acetaminophen toxicity. Drug Dev Res. 2015;76(5):251-8.

55. Bateman DN, Dear JW, Thanacoody HK, Thomas SH, Eddleston M, Sandilands EA, et al. Reduction of adverse effects from intravenous acetylcysteine treatment for paracetamol poisoning: A randomised controlled trial. Lancet. 2014;383 (9918):697-704.

56. Mehrpour O, Shadnia S, SanaeiZadeh $\mathrm{H}$. Late extensive intravenous administration of $\mathrm{N}$-acetylcysteine can reverse hepatic failure in acetaminophen overdose. Hum Exp Toxicol. 2011;30(1):51-4.

57. Mahmoudi GA, Astaraki P, Mohtashami AZ, Ahadi M. $\mathrm{N}$-acetylcysteine overdose after acetaminophen poisoning. Int Med Case Rep J. 2015;8:65-9.

58. Blackford MG, Felter T, Gothard $\mathrm{MD}$, Reed MD. Assessment of the clinical use of intravenous and oral $\mathrm{N}$ acetylcysteine in the treatment of acute acetaminophen poisoning in children: 
A retrospective review. Clin Ther. 2011;33(9):1322-30.

59. Srinivasan V, Corwin D, Verceles AC. An accidental overdose of $\mathrm{N}$ acetylcysteine during treatment for acetaminophen toxicity. Clin Toxicol (Phila). 2015;53(5):500.

60. Buckley NA, Dawson AH, Isbister GK. Treatments for paracetamol poisoning. BMJ. 2016;353:i2579.

61. Lucyk SN, Hoffman RS, Nelson LS, Fuentes M, Tavangarian K. Challenges with AST/ALT ratio in acetaminophen poisoning. Clin Toxicol (Phila). 2015;53(7):786.

62. Duffull SB, Isbister GK. Predicting the requirement for $\mathrm{N}$-acetylcysteine in paracetamol poisoning from reported dose. Clin Toxicol (Phila). 2013;51(8):772-6.

63. Abirami A, Nagarani G, Siddhuraju P. Hepatoprotective effect of leaf extracts from Citrus hystrix and C. Maxima against paracetamol induced liver injury in rats. Food Sci Human Well. 2015;4:35-41.

64. Kaplowitz N, Win S, Than TA, Liu ZX, Dara L. Targeting signal transduction pathways which regulate necrosis in acetaminophen hepatotoxicity. J Hepatol. 2015;63(1):5-7.

65. Karadas S, Aslan M, Gonullu H, Kati C, Duran L, Olmez S, et al. Acetaminophen intoxication is associated with decreased serum paraoxonase and arylesterase activities and increased lipid hydroperoxide levels. Hum Exp Toxicol. 2014;33(11):1134-40.

66. Alotaibi SA, Alanazi A, Bakheet SA, Alharbi NO, Nagi MN. Prophylactic and therapeutic potential of acetyl1-carnitine against acetaminopheninduced hepatotoxicity in mice.
J Biochem Mol Toxicol. 2016;30(1):5-11.

67. Carthy ER, Ellis SD. Paracetamol poisoning in the UK: a meeting report from Pharmacology 2013. Expert Rev Clin Pharmacol. 2014;7(2):147-9. 